\title{
Overlap in genomic variation associated with milk fat composition in Holstein Friesian and Dutch native dual-purpose breeds
}

\author{
M. H. T. Maurice-Van Eijndhoven, ${ }^{\star} \dagger$ H. Bovenhuis, $\dagger$ R. F. Veerkamp,${ }^{*} \dagger$ and M. P. L. Calus ${ }^{* 1}$ \\ *Animal Breeding and Genomics Centre, Wageningen UR Livestock Research, PO Box 338, 6700 AH Wageningen, the Netherlands \\ †Animal Breeding and Genomics Centre, Wageningen University, PO Box 338, $6700 \mathrm{AH}$ Wageningen, the Netherlands
}

\begin{abstract}
The aim of this study was to identify if genomic variations associated with fatty acid (FA) composition are similar between the Holstein-Friesian (HF) and native dual-purpose breeds used in the Dutch dairy industry. Phenotypic and genotypic information were available for the breeds Meuse-Rhine-Yssel (MRY), Dutch Friesian (DF), Groningen White Headed (GWH), and HF. First, the reliability of genomic breeding values of the native Dutch dual-purpose cattle breeds MRY, DF, and GWH was evaluated using single nucleotide polymorphism (SNP) effects estimated in HF, including all SNP or subsets with stronger associations in HF. Second, the genomic variation of the regions associated with FA composition in HF (regions on Bos taurus autosome 5, 14, and 26), were studied in the different breeds. Finally, similarities in genotype and allele frequencies between MRY, DF, GWH, and HF breeds were assessed for specific regions associated with FA composition. On average across the traits, the highest reliabilities of genomic prediction were estimated for GWH (0.158) and DF (0.116) when the 8 to $22 \mathrm{SNP}$ with the strongest association in HF were included. With the same set of SNP, GEBV for MRY were the least reliable (0.022). This indicates that on average only 2 (MRY) to $16 \%$ $(\mathrm{GWH})$ of the genomic variation in $\mathrm{HF}$ is shared with the native Dutch dual-purpose breeds. The comparison of predicted variances of different regions associated with milk and milk fat composition showed that breeds clearly differed in genomic variation within these regions. Finally, the correlations of allele frequencies between breeds across the 8 to $22 \mathrm{SNP}$ with the strongest association in HF were around 0.8 between the Dutch native dual-purpose breeds, whereas the correlations between the native breeds and HF were clearly lower and around 0.5. There was no consistent relationship between the reliabilities of genomic prediction for a specific breed and the correlation between the allele
\end{abstract}

Received December 5, 2014.

Accepted June 2, 2015.

${ }^{1}$ Corresponding author: mario.calus@wur.nl frequencies of this breed and HF. In conclusion, most of the genomic variation associated with FA composition in the Dutch dual-purpose breeds appears to be breedspecific. Furthermore, the minor allele frequencies of genes having an effect on the milk FA composition in HF were shown to be much smaller in the breeds MRY, $\mathrm{DF}$, and GWH, especially for the MRY breed.

Key words: cattle breeds, milk, fatty acid, genomic variation

\section{INTRODUCTION}

Over the last few decades, a clear change in the dairy industry has taken place toward high production, especially in industrialized countries. High emphasis on selection for milk yield and the consolidation and globalization of breeding companies has led to professional breeding programs for specialized dairy cattle breeds (Brotherstone and Goddard, 2005; Shook, 2006). This development has led directly to a decline in use of native dual-purpose breeds, which have been used for milk and beef production. For example, in the Netherlands the percentage of dairy cows in dairy industry belonging to Dutch native dual-purpose breeds declined from 91.3 to $1.4 \%$ in the last $30 \mathrm{yr}$. This reduction in breed variability has led to a decrease of genetic variability in the total cattle population as individual cattle breeds comprise unique genetic variation (European Cattle Genetic Diversity Consortium, 2006). In this regard, safeguarding existing genetic variation can be important to be able, in the future, to exploit currently unknown genetic variation (Oldenbroek, 2007). In addition, from the perspective of breeding programs, it is important to know the magnitude of genetic variation between breeds and, more concretely, whether genomic prediction performed in Holstein-Friesian (HF) can be used in the numerically small native dual-purpose breeds. Therefore, it is of interest for the dairy cattle industry to know whether the genetic variation in native dual-purpose breeds differs from the HF breed. One trait, which is extensively studied in the HF breed and increasingly more important for the dairy industry, is the FA composition in milk (e.g., Soyeurt et al., 2006; 
Stoop et al., 2008). The interest of the dairy industry in the possibilities to modify FA composition in milk arises from supposed associations of milk FA composition with human health (e.g., Palmquist et al., 2006; Astrup et al., 2011) and milk processability (e.g., Smet et al., 2009). In addition to genetic variance for milk FA composition observed within breeds, differences in milk FA composition among dairy cattle breeds are also described in several studies (e.g., Soyeurt et al., 2011; Maurice-Van Eijndhoven et al., 2013a).

Studying the relation between the bovine genome and the FA composition in bovine milk is an important step toward understanding the genetic variability of milk FA composition. For example, on the bovine genome in 2 genes, namely diacylglycerol acyltransferase 1 (DGAT1) and stearoyl-CoA desaturase 1 (SCD1), polymorphisms are reported to have large associations with milk FA composition of HF cattle (e.g., Bouwman et al., 2011; Bernard et al., 2013). To investigate the genetic variability in native dual-purpose breeds, one possible approach is to compare whether different genomic regions have a similar contribution to trait variation in different cattle breeds. Allele frequencies in DGAT1 and SCD1 polymorphisms have been reported in several breeds (Spelman et al., 2002; Kgwatalala et al., 2007). For example, different allele frequencies of the DGAT1 232K allele in New Zealand HF (0.6), Ayrshire (0.22), and Jersey (0.88) were reported by Spelman et al. (2002) and of the SCD1 293A allele in Canadian HF (0.83) and Jersey (0.95) are reported by Kgwatalala et al. (2007). Therefore, an appropriate question is whether effects of genomic regions on the milk fat composition of HF cows are similar for the native dual-purpose breeds.

The aim of the current study is to investigate similarities in genomic variation associated with milk FA composition in the HF breed and native Dutch dualpurpose breeds. This will be achieved by evaluating the reliability of predicted genomic breeding values for fat composition in milk of the native Dutch dual-purpose cattle breeds Meuse-Rhine-Yssel (MRY), Dutch Friesian (DF), and Groningen White Headed (GWH) using SNP effects estimated in $\mathrm{HF}$ and by comparing the genomic variability in regions on the genome showing large effects on milk FA composition.

\section{MATERIALS AND METHODS}

\section{Data Collection}

Phenotypic and genotypic data were collected in 3 different periods respectively on 2,500 and 1,867 cows belonging to 4 different breeds (Table 1). The complete data set contains 1 milk sample for each cow that was analyzed using GC-MS or mid-infrared spectrometry (MIR), and genotyped animals are distributed differently across periods. In detail, milk samples of the first sample period were collected within the project of the Dutch Milk Genomics Initiative between May and June 2005. The GC-MS profiles were obtained from morning milk samples from 1,811 cows. Blood samples were also collected for genotyping. All cows belong to the HF breed and were sampled during the first parity between 97 and 355 DIM and each herd-test-day (HTD) comprises a minimum of 3 records. Detailed information on this data set is reported in previous studies, where it is referred to as the data representing summer milk (Rutten et al., 2009; Bouwman et al., 2012; Duchemin et al., 2013).

Data of the second sample period was collected during December 2008 and March 2009. The GC-MS profiles of morning milk samples were collected from 137 cows, and 79 of them had good-quality DNA samples available and were genotyped. Cows belonged to 3 breeds: MRY (50 samples from 3 herds), DF (44 samples from 3 herds), and GWH (43 samples from 3 herds). Parity of the cows varied between 1 and 9 and DIM varied between 5 and 535 . Detailed information on these GCMS data are reported in Maurice-Van Eijndhoven et al. (2011). An overview of the data is shown in Table 1.

Data of the third sample period was collected during the end of August 2011 and the beginning of November 2011. The GC-MS or MIR profiles of morning milk samples were collected from 552 cows and 280 of them were also genotyped (Table 1 ). All cows were purebreds belonging to the breeds MRY, DF, GWH, or HF (i.e., they were registered with at least $87.5 \%$ of the genes of any of the breeds mentioned). The data collected in 2011 contains 3 herds with milk samples of multiple breeds (one herd with $28 \mathrm{MRY}$ and $15 \mathrm{HF}$ cows; one herd with $6 \mathrm{GWH}$ and $36 \mathrm{HF}$ cows; and one herd with 5 MRY, 33 DF, and 4 GWH cows). The other 11 herds included in the study used a single breed: $242 \mathrm{MRY}$ cows on 3 herds, 94 DF cows on 2 herds, 136 GWH cows on 3 herds, and $80 \mathrm{HF}$ cows on 3 herds.

\section{Measuring FA Composition}

All milk samples were treated immediately with $0.03 \%$ (wt/wt) sodium azide to avoid microbiological growth. To obtain detailed FA compositions, all milk samples were analyzed at the laboratory of Qlip N.V. (Zutphen, the Netherlands) by GC-MS or by Fourier-transform interferogram machines (MilkoScan FT 6000, Foss Electric, Hillerød, Denmark) to obtain MIR profiles. The GC-MS outputs were generated by analyzing methyl esters. Fatty acid methyl esters were prepared using fat fractions extracted from the milk, 
Table 1. Overview of the number of animals per breed and fixed effects per sample period

\begin{tabular}{|c|c|c|c|c|}
\hline \multirow[b]{2}{*}{ Item } & \multicolumn{4}{|c|}{ Data } \\
\hline & Data $2005^{1}$ & Data $2008-2009^{1}$ & Data $2011^{1}$ & Data $2011^{2}$ \\
\hline Number of animals & 1,811 & 137 & 319 & 233 \\
\hline \multicolumn{5}{|l|}{ Breed, $\mathrm{n}$} \\
\hline Meuse-Rhine-Yssel & 0 & 50 & 144 & 98 \\
\hline Genotyped $^{3}$ & 0 & 39 & 106 & 0 \\
\hline Groningen Whited Headed & 0 & 43 & 105 & 31 \\
\hline Genotyped $^{3}$ & 0 & 25 & 89 & 0 \\
\hline Dutch Friesian & 0 & 44 & 70 & 24 \\
\hline Genotyped $^{3}$ & 0 & 15 & 56 & 0 \\
\hline Holstein-Friesian & 1,811 & 0 & 0 & 80 \\
\hline Genotyped & 1,811 & 0 & 0 & 0 \\
\hline Total with phenotypes & 1,544 & 137 & 319 & 233 \\
\hline Total with genotypes & 1,811 & 79 & 280 & 0 \\
\hline \multicolumn{5}{|l|}{ Fixed effects, average (SD) } \\
\hline DIM & $274(42)$ & $144(88)$ & $158(94)$ & $162(98)$ \\
\hline Parity $^{4}$ & $1.0(0)$ & $2.8(2.0)$ & $2.7(1.8)$ & $3.2(1.9)$ \\
\hline $\mathrm{Age}^{5}$ & $26(2)$ & $52(28)$ & $50(23)$ & $56(25)$ \\
\hline \multicolumn{5}{|c|}{${ }^{1}$ FA composition based on GC-MS. } \\
\hline \multicolumn{5}{|c|}{${ }^{2}$ FA composition based on mid-infrared spectroscopy. } \\
\hline \multicolumn{5}{|c|}{${ }^{3}$ For all genotyped cows of the breeds, GC-MS phenotypes were also available. } \\
\hline \multicolumn{5}{|c|}{$\begin{array}{l}{ }^{4} \text { Parity was divided in } 4 \text { classes for corresponding lactation numbers of parity } 1,2 \text {, and } 3 \text { and the } 4 \text { th cla } \\
\text { included parity } 4-10 \text {. }\end{array}$} \\
\hline
\end{tabular}

as described in ISO Standard 15884 (ISO-IDF, 2002b). Methyl esters were analyzed, as described in ISO Standard 15885 (ISO-IDF, 2002a), according to the 100\% FAME method with a $100-\mathrm{m} \times 0.25$-mm polar column (Select Fame Varian CP 7420, Varian Inc., Palo Alto, CA).

For the final analyses, fat percentage, 1 group of FA, and 9 individual FA were chosen: short-chain FA (C4:0-C12:0); C12:1 cis-9; C14:0; C14:1 cis-9; C16:0; C18:0; C18:1 trans-6, 9, 11; C18:1 cis-9; C18:2 cis-9, 12 and C18:2 cis-9,trans-11. The group of FA and individual FA were analyzed as grams per deciliter of milk. The FA were chosen for (1) their reasonably high correlations between summer and winter milk (Duchemin et al., 2013), (2) their biological background (generated through de novo synthesis or by $\Delta^{9}$-desaturases; Schennink et al., 2008; Soyeurt et al., 2008), and (3) for the (lack of) relation with DGAT1 or SCD1 (Schennink et al., 2008).

\section{Genotype Data}

The breeds MRY, DF, and GWH were genotyped using the Illumina high-density (HD) SNP chip (Illumina, San Diego, CA) and the HF breed with a custom 50k SNP chip (Illumina Inc.), which was imputed to HD SNP data. For all breeds, genotypes were edited for call rates and minor allele frequency. Missing HD genotypes were imputed for MRY, DF, and GWH, and for HF all HD SNP not included on the 50k chip were imputed. Additional editing steps were partly different, as explained below, because data were obtained from multiple studies.

In total, 359 animals were genotyped for the breeds MRY, DF, and GWH. Genotypes with a GC-MS score $<0.2$ were set to missing. After this initial editing step, we removed SNP with a call rate $<85 \%$, a minor allele frequency $<0.02$, unknown map position, known position on the sex chromosomes, or when not all 3 genotypes were observed. Removing SNP for which 3 genotypes were not observed is similar to removing SNP based on a commonly applied Hardy-Weinberg test aiming to remove only the most extreme outlier SNP and not those that depart from Hardy-Weinberg equilibrium due to selection (Wiggans et al., 2009). Further edits included removing animals with a call rate $<85 \%(\mathrm{n}=8)$, parent-offspring inconsistencies $(\mathrm{n}=2)$, and because, based on principal component analysis of the genotype data, animals clustered with a different breed than registered by the herd book registration (n $=1$ ). Finally, missing genotypes were imputed using Beagle (Browning and Browning, 2007). After these edits, 619,056 segregating SNP were available.

The HF data contained 1,684 animals with imputed HD genotypes for 734,393 SNP. Those animals were initially genotyped with a custom 50k SNP chip. The HD genotypes were imputed using HD genotypes of all 55 sires of the HF cows, combined with HD genotypes of another 1,278 HF bulls available at CRV (Arnhem, the Netherlands). More details on these (imputed) 
genotypes are provided by Bouwman et al. (2014). Before combining them with the other data set, SNP with a minor allele frequency $<0.02$ or when not all 3 genotypes were observed were removed, leaving 602,445 SNP.

The imputed HD SNP data for the HF animals was combined with the HD SNP data of the other breeds, yielding a combined data set consisting of 571,275 SNP, which met the editing criteria in both data sets. In the combined data set, from each pair of neighboring SNP with an $\mathrm{r}^{2}$ (linkage disequilibrium) value of 1.0, one of the SNP was removed. After this final step, 350,207 SNP were left in the data set used for analysis.

\section{Statistical Analysis: Correcting Phenotypes for Fixed Effects}

Before estimating SNP effects, the record of each cow was corrected for the fixed effects of DIM, parity, age at calving, HTD, breed, and method of measuring the FA using the following model (using ASReml 3.0, Gilmour et al., 2009):

$$
\begin{gathered}
\mathrm{y}_{\mathrm{ijklmno}}=\mu+\mathrm{b}_{1} \times \text { DIM }_{\mathrm{i}}+\mathrm{b}_{2} \times \exp ^{-0.05 \times \text { DIM }} \\
+ \text { parity }_{\mathrm{j}}+\mathrm{b}_{3} \times \operatorname{age}_{\mathrm{k}}\left(\text { parity }_{\mathrm{j}}\right)+\mathrm{HTD}_{\mathrm{l}} \\
+\operatorname{breed}_{\mathrm{m}}+\operatorname{method}_{\mathrm{n}}+\mathrm{e}_{\mathrm{ijklmno}}
\end{gathered}
$$

where $y_{\mathrm{ijklmno}}$ was the dependent variable for cow o in DIM $\mathrm{i}$, with parity $\mathrm{j}$, age at calving $\mathrm{k}$, producing at HTD l, belonging to breed $\mathrm{m}$, and with FA composition obtained using method $\mathrm{n}$. The $\mu$ was the overall mean of the model; $b_{1}$ and $b_{2}$ were the fixed regression coefficients on $\mathrm{DIM}_{\mathrm{i}}$ modeled with a Wilmink curve
(Wilmink, 1987); parity was $_{\mathrm{j}}$ the fixed effect with 4 classes for corresponding lactation numbers of parity 1,2 , and 3 and the fourth class included parity 4 to $10 ; b_{3}$ was the fixed regression coefficient on age at calving (age $\mathrm{k}_{\mathrm{k}}$, days) within the jth parity; $\mathrm{HTD}_{1}$ was the effect defining groups of cows sampled in the same herd on the same sample date; breed $_{\mathrm{m}}$ was the effect defining groups of cows belonging to the same breed (MRY, GWH, DF, or HF); method $_{n}$ was the effect with 2 classes corresponding for the method used to obtain the FA composition (GC-MS or MIR); and $e_{\mathrm{ijklmno}}$ is the random residual effect. Predicted FA composition based on MIR profiles was included for 272 animals without genotypes as y variate in the analysis to obtain more precise correction of the phenotypes for the fixed effects. Using MIR the majority of FA can be predicted with moderate $\left(\mathrm{R}^{2}=0.60\right.$ to 0.80 for $\mathrm{C} 14: 0$ cis-9, C16:0 cis-9, and $\mathrm{C} 18: 0)$ to high $\left(\mathrm{R}^{2} \geq 0.80\right.$ for the individual FA C4:0-C16:0 and groups of FA) accuracy (MauriceVan Eijndhoven et al., 2013b). The residuals of this model for the records obtained with GC-MS were used as corrected phenotypes in the subsequent genomic analyses. Table 2 shows an overview of the corrected phenotypes per sample period.

\section{Statistical Analysis: Estimation of SNP Effects}

To identify if similarities existed in genomic variation associated with milk FA composition, the fat composition in milk of Dutch cattle breeds MRY, DF, and GWH was predicted using SNP effects estimated based on the 1,544 HF cows. For this part of the analysis, genotype data and the residuals obtained from the

\begin{tabular}{|c|c|c|c|c|}
\hline Item & \multicolumn{4}{|c|}{ Year(s) } \\
\hline Fat\% & $4.26(1.25)$ & $4.56(1.29)$ & $4.55(1.39)$ & $4.36(1.54)$ \\
\hline C4:0-C10:0 & $0.4219(0.1498)$ & $0.5085(0.1698)$ & $0.4667(0.1470)$ & $0.4349(0.1556)$ \\
\hline $\mathrm{C} 12: 1$ & $0.0046(0.0024)$ & $0.0043(0.0029)$ & $0.0047(0.0031)$ & $0.0056(0.0024)$ \\
\hline $\mathrm{C} 16: 0$ & $1.2494(0.4813)$ & $1.3812(0.4852)$ & $1.3336(0.4852)$ & $0.9825(0.6584)$ \\
\hline C18:0 & $0.4234(0.1903)$ & $0.4782(0.1877)$ & $0.4406(0.2314)$ & $0.3446(0.1891)$ \\
\hline C18:1 cis-9 & $0.0680(0.1333)$ & $0.0589(0.1399)$ & $0.0755(0.1889)$ & $0.0873(0.1807)$ \\
\hline $\mathrm{C} 18: 1$ trans- $6,9,11$ & $0.8596(0.1783)$ & $0.7596(0.1739)$ & $0.8732(0.2328)$ & $0.9456(0.2413)$ \\
\hline $\mathrm{C} 18: 2$ cis $-9,12$ & $0.0472(0.0170)$ & $0.0593(0.0264)$ & $0.0538(0.0259)$ & $0.0591(0.0206)$ \\
\hline C18:2 cis-9,trans-11 & $0.0232(0.0142)$ & $0.0182(0.0086)$ & $0.0273(0.0228)$ & $0.0350(0.0161)$ \\
\hline
\end{tabular}
model to precorrect the phenotypes were used. The

Table 2. Overview of the corrected phenotypes per sample period

${ }^{1}$ Milk samples analyzed using GC-MS.

${ }^{2}$ Milk samples analyzed using mid-infrared spectroscopy.

${ }^{3}$ Corrected phenotypes for fat percentage (the production trait fat percentage was analyzed using mid-infrared spectroscopy for all data) and individual FA and groups of FA in grams per deciliter of milk. 
model used is commonly known as BayesC (Habier et al., 2011), and is described by

$$
\mathbf{y}=\mathbf{1} \mu+\mathbf{Z u}+\mathbf{X} \alpha+\mathbf{e}
$$

where $\mathbf{y}$ contains precorrected phenotypes, $\mathbf{1}$ is a vector of ones, $\mu$ is the overall mean, $\mathbf{Z}$ is an incidence matrix that links records to individuals, u contains random polygenic effects of all individuals, $\mathbf{X}$ is a matrix that contains the scaled and centered genotypes of all individuals, $\alpha$ contains the (random) allele substitution effects for all loci, and e contains the random residuals (see Technical Appendix; http://dx.doi.org/10.3168/ jds.2014-9196). Polygenic effects were included in the model because the HF data contained only a limited number of sires with relative large groups of daughters. Omitting a polygenic effect with such a data structure is expected to lead to spurious associations (e.g., Balding, 2006) that will not have any predictive value for the other breeds.

From the output of the BayesC model, a Bayes Factor $(\mathbf{B F})$ was calculated for each SNP as

$$
\mathrm{BF}=\frac{\operatorname{Pr}\left(H_{1} \mid y\right)}{1-\operatorname{Pr}\left(H_{1} \mid y\right)} \div \frac{\operatorname{Pr}\left(H_{1}\right)}{1-\operatorname{Pr}\left(H_{1}\right)},
$$

where $H_{1}$ is the hypothesis that the marker has a nonzero effect; $\operatorname{Pr}\left(H_{1} \mid y\right)$ is the posterior probability of the hypothesis and $\operatorname{Pr}\left(H_{1}\right)$ is the prior probability of the hypothesis; and $1-\operatorname{Pr}\left(H_{1} \mid y\right)$ and $1-\operatorname{Pr}\left(H_{1}\right)$ represent the posterior and prior probability for the alternative hypothesis, respectively. Bayes Factors are an indication of the size of the effects SNP have on the trait analyzed. High BF indicates that a SNP is likely to be associated with a QTL affecting the trait. To give an overview of SNP having an effect on the different milk traits in HF, a Manhattan plot for the BF for each SNP was produced. Plotted BF were smoothed by calculating for each SNP the average of its own BF and those of the 4 SNP located on either side.

\section{Statistical Analysis: Estimation of Genomic Estimated Breeding Values}

The SNP effects estimated using the data set of the HF population sampled in 2005 were used to calculate genomic estimated breeding values (GEBV) for the genotyped cows of the breeds MRY, DF, and GWH sampled in 2008 to 2009 and 2011. To check how valid the GEBV are using SNP effects estimated in HF, reliabilities (i.e., accuracies squared) of the predicted breeding values were calculated. The reliability can be expressed as:

$$
\mathrm{r}_{g \hat{g}}^{2}=\mathrm{r}_{y \hat{g}}^{2} / \mathrm{h}^{2}
$$

which follows Verbyla et al. (2010), where $\mathrm{r}_{y \hat{g}}^{2}$ is the squared correlation between the observed phenotype in MRY, DF, or GWH and their GEBV, and $\mathrm{h}^{2}$ is the heritability of the trait. The $\mathrm{h}^{2}$ used were estimated for a Dutch MRY population (Maurice-Van Eijndhoven et al., 2014; Supplementary Table S1; http://dx.doi. org/10.3168/jds.2014-9196) and used for all 3 breeds (MRY, DF, and GWH) because no $\mathrm{h}^{2}$ estimates were available for DF and GWH. Heritabilities for MRY for the individual FA C12:1 to C18:0 ranged from 0.23 to 0.34, for the individual FA C14:1 cis-9 was 0.27 , for the individual $\mathrm{C} 18$ unsaturated FA ranged from 0.10 to 0.26, and for the short-chain group of FA C4:0 to C10:0 was 0.27 . For $\mathrm{C} 12: 1$ and $\mathrm{C} 18: 1$ trans- $6,9,11$ no estimates were available for $h^{2}$ for MRY. To enable approximation of the accuracies for those $2 \mathrm{FA}$, values of respectively 0.3 and 0.16 were used, resembling to $h^{2}$ estimates obtained in MRY for FA that were shown to have similar variance as for C12:1 and C18:1 trans-6, 9, 11 in HF. Because the same $h^{2}$ estimates were used for all breeds, this only allowed to approximate accuracies of GEBV instead of predicted phenotypes, although it did not allow us to differentiate between the breeds. The GEBV were calculated using all SNP or only SNP having an estimated effect in HF with different thresholds of BF (greater than 10,100, or 1,000). This allows us to investigate the effect of regions with greater effects on FA composition in HF on the accuracy of prediction in the other breeds. To further evaluate similarities between breeds in those genomic regions, allele frequencies were compared, as well as variance explained per SNP. Finally, the percentage of the genetic variation explained by the SNP for each breed was computed as $2 p q \hat{\alpha}^{2} / \operatorname{var}(a)$, where $\operatorname{var}(a)$ was the genetic variance for HF calculated by $\operatorname{var}(a)=\mathrm{h}^{2} \times \operatorname{var}(y)$, where $y$ were precorrected phenotypes and the $\mathrm{h}^{2}$ for HF were obtained from Maurice-Van Eijndhoven et al. (2014). For trait C18:2 cis-9,trans-11, no SNP were found with a $\mathrm{BF}>1,000$; therefore, this trait was not included in this last evaluation. To further evaluate similarities between breeds in those genomic regions, allele frequencies for the different scenarios were compared.

To allow comparison of the reliabilities obtained for the other breeds, reliabilities within HF were computed as well. This involved a random 10 -fold cross validation, where all $\mathrm{HF}$ animals were randomly allocated to 10 equally sized folds. For each of the folds, GEBV were predicted using the other 9 folds as training data. Here, the same BayesC model including polygenic effects was applied and polygenic effects were not used for the 
prediction, similar as for other breeds. Heritabilities, required to compute the reliabilities, were obtained from (Maurice-Van Eijndhoven et al., 2014).

\section{RESULTS}

\section{Corrected Data}

Cows sampled in 2005 were on average 25 mo old at calving, sampled at 274 DIM, and were all in their first parity (Table 1). Cows sampled in 2008 to 2009 and 2011 were older, being on average 49 to 55 mo old at calving, sampled at 144 to 162 DIM, and in their third parity. An overview of the data, after correction for fixed effects, is given in Table 2. The data are grouped per sample period to enable comparison of properties across the underlying data sets. Fat percentage was, on average, highest for data collected in 2008 to 2009 (4.56\%), lowest for data collected in 2005 (4.26\%), and most variable for data collected in 2011 $(\mathrm{SD}=1.39-1.54)$. The average contents in milk of all saturated FA (C4:0-C10:0; C14:0; C16:0; and C18:0) of the samples collected in 2008 to 2009 were higher and all unsaturated FA contents, except C18:2 cis-9, 12 , were lower compared with the samples collected in 2005 and 2011. The average contents of unsaturated FA, except C18:2 cis-9, 12, were generally lowest in the data collected in 2011, which was analyzed using MIR. Nevertheless, the standard deviations were comparable for all periods.

\section{SNP Effects: Reliabilities of GEBV}

Figure 1 gives an overview of the BF of all SNP across the genome for each of the considered traits. Several peaks are observed for the BF across multiple traits, indicating several QTL that are associated with the FA traits included in our study. As shown in Table 3, between 2,405 and 4,734 SNP showed a BF above the threshold of 10, between 46 and 311 SNP above 100, and between 0 and 22 SNP above 1,000.

The reliabilities of GEBV of fat content ranged from 0.158 to 0.193 for $\mathrm{GWH}$ and from 0.237 to 0.256 for DF when SNP effects were used with a BF >10; no clear improvement was observed when using SNP with a $\mathrm{BF}>100$ or $>1,000$ (Table 4). For MRY, the reliabilities for total fat percentage were clearly lower (between 0.034 and 0.046) and no clear improvement was observed when using SNP with a BF $>100$ or $>1,000$. The GEBV of the FA C12:1 showed relatively high reliabilities for GWH $(0.397-0.492)$ and for DF around 0.150 when considering only SNP with a BF $>10$. For the FA C14:0 and C16:0, the reliabilities of the GEBV for GWH were all above 0.2 when consider- ing only SNP with a $\mathrm{BF}>10$. The highest reliability (0.758) was observed when using all SNP for GWH and C14:1 cis-9. However, the reliability dropped almost to zero when only SNP were considered with a $\mathrm{BF}>10$. For the FA C18:1 cis-9, the reliability of the GEBV for DF were relatively high $(0.182-0.185)$, regardless the possible threshold based on BF, whereas for MRY and GWH the reliabilities were almost zero. The reliabilities of the GEBV for MRY were below 0.1 for all traits, except for C14:1 cis-9 using all 305,207 SNP effects to calculate the GEBV (0.178); however, when considering only SNP with a $\mathrm{BF}>10$, this reliability dropped toward zero.

Of all 33 traits by breed combinations, 10, 11, and 12 had reliabilities greater than 0.05 when using SNP with $\mathrm{BF}>10,>100$, or $>1,000$, respectively (Table 4). Reliabilities of the GEBV for HF were in general considerably higher than for the other breeds, only in a few cases the reliabilities for the other breeds were higher than the HF reliabilities.

Overall, the reliabilities of GEBV clearly differed between using all 350,207 SNP and when considering only SNP with a $\mathrm{BF}>10,>100$, or $>1,000$, whereas between subsets of SNP with the different BF thresholds the reliabilities of the GEBV were very similar. The estimation of SNP effects in HF have very low predictive ability in MRY (on average 0.022 with a BF $>1,000$ ) and some clear predictive ability in DF and GWH, although the realized reliabilities were on average low, being 0.116 for DF and 0.158 for GWH.

\section{SNP Variances}

Table 5 shows the predicted variances explained per SNP for the SNP whose variance in at least 1 of the breeds was $\geq 1 \%$ relative to the total genetic variance in the HF breed. The region of DGAT1 on BTA14 was found to have the largest effect on most of the traits. This region explained large parts of the genetic variance in $\mathrm{HF}$ for the traits fat percentage, with $\mathrm{C} 4: 0$ to C10:0, C12:1, C14:0, C14:1 cis-9, C16:0, C18:0, and C18:2 cis-9, 12 ranging from 1.1 (C18:2 cis-9, 12) to 19.0\% (C16:0). For the breeds DF and GWH, the region of DGAT1 was also found having an effect for several traits (ranging from $0.2 \%$ for $\mathrm{C} 18: 2$ cis-9, 12 to $3.3 \%$ for C16:0 for GWH and from zero effect for 6 traits to $2.2 \%$ for C16:0 for DF), although heavily reduced in comparison with $\mathrm{HF}$. Almost no effect of the genomic region on BTA14 was found for the traits in MRY. On BTA26, a genomic region was found having an effect on the genetic variance of the traits C12:1 and $\mathrm{C} 14: 1$ cis-9 for all breeds, which was largest for $\mathrm{HF}$ and DF (explaining 2.8\% of C12:1 in HF milk; $8.0 \%$ of $\mathrm{C} 12: 1$ in DF; $2.6 \%$ of $\mathrm{C} 14: 1$ cis-9 in $\mathrm{HF} ; 7.4 \%$ of $\mathrm{C} 14: 1$ 


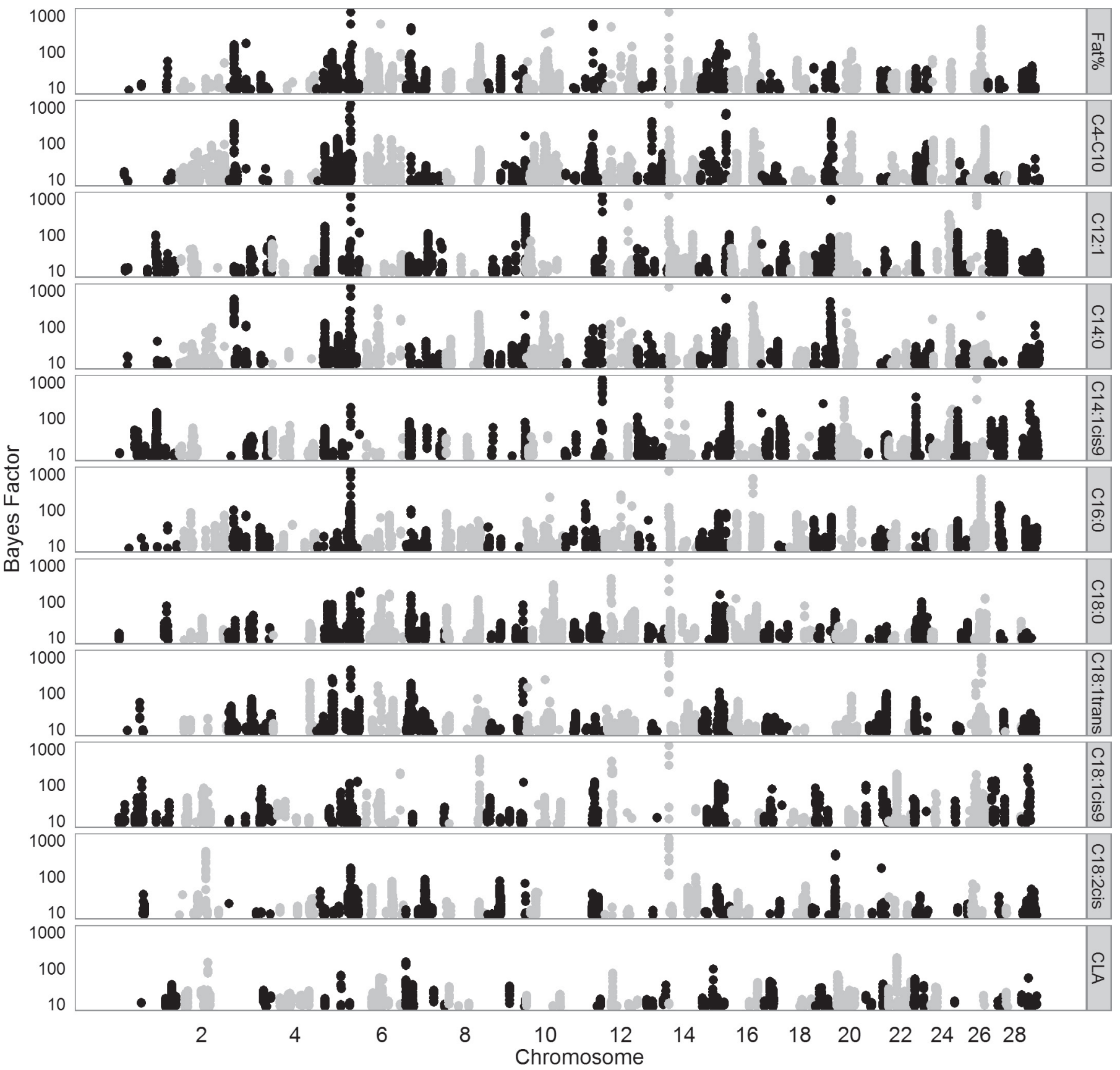

Figure 1. Manhattan plot showing the Bayes Factors for each SNP having an effect on the bovine milk FA in Holstein-Friesians. Plotted Bayes Factors were the average of the SNP itself and those of the 4 SNP located on either side. C18:1 trans = sum of C18:1 trans-6, C18:1 trans-9, and C18:1 trans-11; C18:2 cis = C18:2 cis-9,12; CLA = C18:2 cis-9,trans-11.

cis-9 in DF of the genetic variance for the whole region, sum of both SNP, respectively). For GWH, this region explained $1.8 \%$ of the variance of the trait $\mathrm{C} 12: 1$ and $5.1 \%$ of $\mathrm{C} 14: 1$ cis-9 and for MRY $1.5 \%$ of $\mathrm{C} 12: 1$ and $4.2 \%$ of $14: 1$ cis-9. On BTA5, one SNP was found with an effect on the genetic variance of the traits fat percentage, for C4:0 to C10:0, C12:1, C14:0, and C16:0 this effect was estimated to explain between 1 and $2 \%$ of the genetic variance for $\mathrm{HF}$, between 0.3 and $0.6 \%$ for DF and MRY, and almost negligible for GWH.

\section{Correlations of Allele Frequencies}

To assess similarities between the breeds across the whole genome, versus in regions associated with FA composition, the correlation of allele frequencies be- 
Table 3. Number of $\mathrm{SNP}^{1}$ with a Bayes Factor (BF) greater than 10, 100 , or 1,000

\begin{tabular}{lccc}
\hline Item & $\mathrm{BF}>10$ & $\mathrm{BF}>100$ & $\mathrm{BF}>1,000$ \\
\hline Fat\%2 & 3,292 & 211 & 14 \\
C4:0-C10:0 & 3,942 & 301 & 13 \\
C12:1 & 3,527 & 255 & 15 \\
C14:0 & 3,852 & 260 & 22 \\
C14:1 cis-9 & 3,140 & 198 & 16 \\
C16:0 & 3,328 & 172 & 14 \\
C18:0 & 3,622 & 170 & 9 \\
C18:1 trans-6,9,11 & 3,593 & 132 & 14 \\
C18:1 cis-9 & 2,405 & 201 & 13 \\
C18:2 cis-9,12 & 2,552 & 80 & 8 \\
C18:2 cis-9,trans-11 & 2,707 & 46 & 0 \\
\hline
\end{tabular}

${ }^{1}$ Number of SNP that exceeded the BF threshold and segregated in Meuse-Rhine-Yssel, Dutch Friesian, or Groningen White Headed.

${ }^{2}$ Fat as percentage and individual FA and groups of FA in grams per deciliter of milk

tween the breeds MRY, DF, GWH, and HF were calculated (Table 6). Across all 350,207 SNP, the correlation was between 0.64 (DF and GWH) and 0.71 (DF and HF as well as MRY and HF; Table 6). When including only SNP with a BF above one of the thresholds for a particular trait, correlations ranged from 0.63 (DF and GWH) to 0.70 (DF and HF as well as MRY and HF) for $\mathrm{BF}>10$; from 0.68 (DF and $\mathrm{GWH}$ ) to 0.72 (DF and HF, MRY and HF, and MRY and DF) for BF $>100$; and from 0.50 (MRY and HF) to 0.83 (MRY and DF) for $\mathrm{BF}>1,000$. For the $\mathrm{FA} \mathrm{C} 18: 1$ trans-6, 9, 11, the correlations of the allele frequencies between the breeds were relatively high (on average 0.80 ) when considering only SNP with a BF >100; however, the reliabilities of the GEBV for this trait were low. A similar trend was found for the FA C14:1 cis-9 showing allele frequencies of on average 0.79 when considering only SNP with a $\mathrm{BF}>1,000$. When considering only SNP with a threshold based on the $\mathrm{BF}>1,000$, it was remarkable that the correlations of the allele frequencies between $\mathrm{HF}$ and the other Dutch breeds were relatively low (on average 0.50-0.53), whereas between the other Dutch breeds MRY, DF, and GWH the correlation was relatively high (on average $0.80-0.83$ ). Considering only SNP with $\mathrm{BF}>10$ or $>100$, yielded correlations between allele frequencies that were very similar to the correlations across all SNP (results not shown). In summary, both the GEBV reliabilities and the correlations between allele frequencies clearly showed differences between the breeds, albeit no consistent relationship between both statistics was noted.

\section{DISCUSSION}

The aim of our study was to identify similarities in genomic variation associated with detailed milk fat composition between the HF breed and native dual-

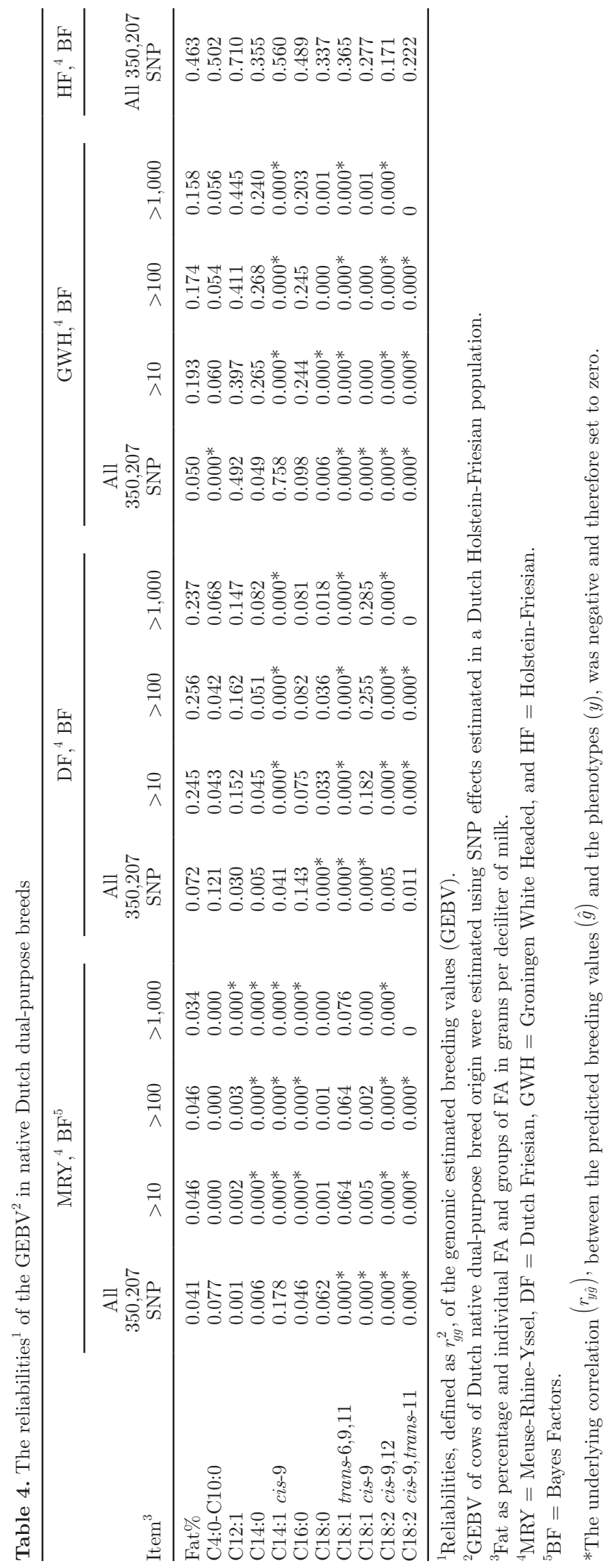

Journal of Dairy Science Vol. 98 No. 9, 2015 
Table 5. The predicted variances explained per SNP with position on the genome for the SNP which explained $\geq 1 \%$ of the genetic variance in at least 1 of the breeds

\begin{tabular}{|c|c|c|c|c|c|c|c|c|}
\hline Trait & SNP name & $\mathrm{BTA}^{1}$ & $\begin{array}{l}\text { Position } \\
\text { (bp) }\end{array}$ & $\begin{array}{l}\text { SNP } \\
\operatorname{var}^{2}\end{array}$ & $\mathrm{HF}^{3,4}$ & $\mathrm{DF}^{3,4}$ & $\mathrm{GWH}^{3,4}$ & $\mathrm{MRY}^{3,4}$ \\
\hline Fat $\%$ & ARS-BFGL-NGS-4939 & 14 & $1,801,116$ & 0.48567 & 13.3 & 1.5 & 2.3 & 0.0 \\
\hline $\mathrm{C} 4: 0-\mathrm{C} 10: 0$ & BovineHD1400000206 & 14 & $1,679,844$ & 0.00654 & 1.3 & 0.0 & 0.3 & 0.2 \\
\hline $\mathrm{C} 12: 1$ & ARS-BFGL-NGS-4939 & 14 & $1,801,116$ & 0.00082 & 10.8 & 1.2 & 1.9 & 0.0 \\
\hline C14:0 & ARS-BFGL-NGS-4939 & 14 & $1,801,116$ & 0.04398 & 9.0 & 1.0 & 1.6 & 0.0 \\
\hline $\mathrm{C} 14: 1$ cis-9 & BovineHD1400000199 & 14 & $1,638,045$ & 0.00192 & 1.6 & 0.2 & 0.9 & 0.3 \\
\hline C16:0 & ARS-BFGL-NGS-4939 & 14 & $1,801,116$ & 0.99652 & 19.0 & 2.2 & 3.3 & 0.0 \\
\hline C18:0 & ARS-BFGL-NGS-4939 & 14 & $1,801,116$ & 0.03338 & 7.5 & 0.9 & 1.3 & 0.0 \\
\hline C18:0 & BovineHD1400000206 & 14 & $1,679,844$ & 0.00751 & 1.7 & 0.0 & 0.4 & 0.3 \\
\hline $\mathrm{C} 18: 0$ & BovineHD1400000204 & 14 & $1,667,797$ & 0.00586 & 1.3 & 0.0 & 0.3 & 0.2 \\
\hline $\mathrm{C} 18: 2$ cis $-9-12$ & ARS-BFGL-NGS-4939 & 14 & $1,801,116$ & 0.00030 & 1.1 & 0.1 & 0.2 & 0.0 \\
\hline $\mathrm{C} 12: 1$ & BovineHD2600005462 & 26 & $21,141,280$ & 0.00026 & 2.8 & 2.6 & 1.8 & 1.4 \\
\hline C16:0 & BovineHD0500026666 & 5 & $93,951,064$ & 0.06288 & 1.2 & 0.3 & 0.2 & 0.5 \\
\hline
\end{tabular}

${ }^{1}$ Bos taurus autosome where SNP is located.

${ }^{2}$ The genetic variance (var) explained by the SNP.

${ }^{3} \mathrm{MRY}=$ Meuse-Rhine-Yssel, DF $=$ Dutch Friesian, GWH $=$ Groningen White Headed, and HF = Holstein Friesian

${ }^{4}$ The percentage of the total genetic variance explained by the SNP for each breed; that is, $\left(2 p q \hat{\alpha}^{2}\right) / \operatorname{var}(a)$, where var $(a)$ is the variance estimated for HF.

${ }^{5}$ SNP was in complete linkage disequilibrium with the SNP located on the base pair positions 21,140,458 and 21,146,794, thus published SNP effect is the accumulated effect of those three SNP in and around the SCD1 gene.

purpose breeds in the Dutch dairy industry. The extent of the overlap in genomic variation between $\mathrm{HF}$ and Dutch dual-purpose breeds was investigated by calculating GEBV for fat composition in the native dualpurpose breeds using SNP effects estimated for HF and by evaluating the underlying SNP effects and variation on the genome within the associated regions. Reli- abilities of GEBV across breeds are reported to be low because linkage disequilibrium (LD) observed within breeds is only partly conserved across breeds (Goddard and Hayes, 2009; Hayes et al., 2009; Hozé et al., 2014). The high-density SNP-chip (Illumina) used in the current study is characterized by short-distance LD that was expected to be maintained across breeds (de Roos

Table 6. The correlations of allele frequencies between breeds ${ }^{1}$ across all SNP, or based only on SNP with a Bayes Factor $>1,000$

\begin{tabular}{|c|c|c|c|c|c|c|c|}
\hline Trait $^{2}$ & $\begin{array}{l}\text { No. of } \\
\text { SNP }^{3}\end{array}$ & $\begin{array}{l}\text { HF and } \\
\text { MRY }\end{array}$ & $\begin{array}{c}\mathrm{HF} \text { and } \\
\mathrm{DF}\end{array}$ & $\begin{array}{c}\text { HF and } \\
\text { GWH }\end{array}$ & $\begin{array}{c}\text { MRY and } \\
\text { DF }\end{array}$ & $\begin{array}{c}\text { MRY and } \\
\text { GWH }\end{array}$ & $\begin{array}{c}\text { DF and } \\
\text { GWH }\end{array}$ \\
\hline - & 350,207 & 0.71 & 0.71 & 0.66 & 0.68 & 0.65 & 0.64 \\
\hline Fat $\%$ & 14 & 0.67 & 0.50 & 0.66 & 0.88 & 0.88 & 0.87 \\
\hline $\mathrm{C} 4: 0-\mathrm{C} 10: 0$ & 13 & -0.21 & 0.29 & 0.44 & 0.79 & 0.63 & 0.94 \\
\hline C12:1 & 15 & 0.62 & 0.22 & 0.22 & 0.83 & 0.86 & 0.90 \\
\hline C14:0 & 22 & 0.40 & 0.57 & 0.41 & 0.65 & 0.62 & 0.76 \\
\hline $\mathrm{C} 14: 1$ cis-9 & 16 & 0.63 & 0.81 & 0.64 & 0.91 & 0.91 & 0.84 \\
\hline C16:0 & 14 & 0.49 & 0.36 & 0.41 & 0.76 & 0.88 & 0.85 \\
\hline C18:0 & 9 & 0.34 & 0.61 & 0.54 & 0.74 & 0.83 & 0.55 \\
\hline C18:1 trans- $6,9,11$ & 14 & 0.39 & 0.42 & 0.54 & 0.90 & 0.94 & 0.89 \\
\hline C18:1 cis-9 & 13 & 0.63 & 0.40 & 0.54 & 0.87 & 0.59 & 0.54 \\
\hline C18:2 cis-9, 12 & 8 & 0.60 & 0.71 & 0.46 & 0.97 & 0.82 & 0.71 \\
\hline C18:2 cis-9,trans-11 & - & - & - & - & - & - & - \\
\hline Average correlation & & 0.50 & 0.52 & 0.53 & 0.83 & 0.80 & 0.80 \\
\hline
\end{tabular}

${ }^{1} \mathrm{MRY}=$ Meuse-Rhine-Yssel, DF $=$ Dutch Friesian, and GWH = Groningen White Headed.

${ }^{2}$ For the traits fat (expressed in percentage) and individual FA and groups of FA (expressed in g/dL milk) the correlations between breeds are given of allele frequencies of SNP with an effect above the thresholds of a Bayes Factor of 1,000.

${ }^{3}$ Number of SNP used to estimate the EBV depends on if the SNP are segregating in the breeds MRY, DF, or GWH. 
et al., 2008). The LD between SNP on the HD SNP chip is indeed being maintained across breeds. However, several empirical studies have shown that the accuracy of across-breed genomic prediction, even when using the HD chip is poor for most traits (Harris et al., 2011; Erbe et al., 2012). Nevertheless, the GEBV reliabilities based on across-breed genomic prediction are expected to be higher for traits with few QTL with large effects, such as fat percentage (e.g., Hayes et al., 2009), and is likely to be higher for FA composition. Thus, if genomic variation associated with detailed milk fat composition in HF were similar to the genomic variation of the native dual-purpose breeds, GEBV reliabilities based on SNP effects estimated in HF were expected to be high. On the contrary, for traits where the genomic variation in HF was very different compared with the other breeds, GEBV reliabilities were expected to be low.

To get a better indication of the effect of individual SNP that have an increasingly larger effect on the traits in HF, GEBV were calculated using different subsets of SNP based on their BF. The highest BF used in this study were $>1,000$, which resulted in a subset of SNP that are very likely to have a strong association with the milk fat composition in HF. The reliabilities of GEBV in our study ranged from 0.000 to 0.758 ; however, reliabilities for the majority of traits were just above zero, indicating large differences in genomic variation associated with detailed milk fat composition between HF and native Dutch dual-purpose breeds. Comparing the native Dutch dual-purpose breeds, the GEBV calculated for the MRY breed were clearly the least reliable whereas for GWH they were most reliable, on average. This indicates that the genetic variation associated with milk fat composition was most different between MRY and HF and least different between GWH and HF.

\section{SNP Effects}

We identified 4 regions having a large effect on the FA composition and explaining more than $1 \%$ of the total genetic variation in the HF breed. Largest effect was found on BTA14 in the region of the DGAT1 gene. Several genome-wide association studies and QTLmapping studies based on the HF breed reported clear associations of the region of DGAT1 on BTA14 with fat percentage and FA composition in the milk of HF cows (e.g., Grisart et al., 2002; Bouwman et al., 2011). The BTA26 harbors the SCD1 gene, which is also reported having an effect on the milk FA composition (e.g., Mele et al., 2007; Schennink et al., 2008; Bouwman et al., 2011). This region was also identified as having an effect on the variability of the content of the FA C12:1 and C14:1 cis-9 in current study. A third region with a clear effect on fat percentage, the group of FA C4:0 to C10:0, and some individual FA was found on BTA 5, where a peak on the Manhattan plot was shown at an SNP at base pair position 93,951,064. This position on BTA5 is close to the microsomal glutathione S-transferase 1 (MGST1) gene, which was found by Wang et al. (2012) to be associated with milk fat percentage.

\section{GEBV, Allele Frequencies, and Genotypes}

In our study, we hypothesized that the allele substitution effects estimated in HF can also be used for the other breeds. As for several traits in the other breeds, the reliability is clearly above 0 when only a small number of SNP are used with high BF; this hypothesis is likely to be valid at least in those cases. In the context of genomic prediction, the analyses performed can be regarded as across-breed genomic prediction, in the sense that the predicted breeds were not included in the reference population used to estimate the SNP effects. Our results suggest that across-breed genomic prediction for some of the traits studied is beneficial. In the literature, empirical studies on across-breed genomic prediction also reported greater-than-zero reliabilities for fat percentage, but generally close to zero reliabilities for other traits (Hayes et al., 2009; Pryce et al. 2011). Reliabilities in the current study based on SNP using $\mathrm{BF}>10,>100$, or $>1,000$ were generally close to those using all SNP. This indicates that adding SNP with, for example, $\mathrm{BF}<10$, merely adds noise rather than a signal with predictive ability. The sampling variance caused by this apparently lead to a few cases where the reliabilities with all SNP were considerably higher than using any of the subsets, and even higher than the HF reliabilities. As indicated, the nonzero reliabilities with high BF mostly resulted from 2 genes with large effect, SCD1 and DGAT1. Therefore, our study provides an example where across-breed genomic prediction is beneficial. However, it should be noted that the results observed here are not generalizable. The traits studied here are partly affected by relatively few QTL with large effect, whereas traits included in current breeding goals are mostly polygenic by nature (Kemper and Goddard, 2012).

No relationship was observed between the correlations of allele frequencies and reliabilities of GEBV. However, we hypothesized that minor allele frequency at regions associated with milk FA composition have an effect on genetic variance explained by SNP and GEBV reliabilities. The minor allele frequencies of genes having an effect on the detailed milk FA composition in HF were shown to be much smaller in the breeds MRY, $\mathrm{DF}$, and GWH, which is especially the case for the MRY breed. These lower minor allele frequencies for 
MRY are the most likely explanation for the near zero observed reliabilities for MRY. This is also consistent with the earlier finding by Maurice-Van Eijndhoven et al. (2014) that the heritabilities for milk FA composition of the MRY breed were clearly smaller than those estimated for HF.

A reasonable explanation for these results could be the small variation in genotypes in the regions where QTL with large effect were found in HF (results are shown in Supplemental Tables S2 and S3; http:// dx.doi.org/10.3168/jds.2014-9196). Despite the limited numbers of genotyped animals of the other breeds, a clear and consistent difference in the frequencies of genotypes was observed in the DGAT1 region for the other breeds compared with HF. High frequencies of the major genotype and almost no cases of the minor genotype were also found in other populations of dualpurpose breeds (e.g., Thaller et al., 2003). Another explanation could be that the genetic variance of fat composition in milk of the Dutch dual-purpose cattle breeds is largely regulated by other and still unknown genomic regions.

\section{CONCLUSIONS}

Estimated SNP effects for FA composition based on HF data had low to no predictive ability in MRY, whereas they did have clear predictive ability for several traits for DF and GWH. The lack of predictive ability in MRY indicates that SNP effects estimated in HF explain less of the genetic variation of the FA composition in milk of MRY compared with the genetic variation of the milk fat composition in DF and GWH. Overall, most of the genomic variation associated with FA composition in the Dutch dual-purpose breeds appears to be breed-specific; however, overlap with variation in $\mathrm{HF}$ on average ranges from very small (MRY) up to $\sim 16 \%$ (GWH) in terms of realized reliabilities using SNP effects estimated in HF. Furthermore, the minor allele frequencies of genes having an effect on the detailed milk FA composition in HF were shown to be much smaller in the breeds MRY, DF, and GWH, which is especially the case for the MRY breed.

\section{ACKNOWLEDGMENTS}

This study was financially supported by the Ministry of Economic Affairs (The Hague, the Netherlands; Programme "Kennisbasis Research," code: KB-04-002-021 and KB-05-003-041). The Dutch Milk Genomics Initiative and the project 'Melk op Maat', funded by Wageningen University (Wageningen, the Netherlands), the Dutch Dairy Association (NZO, Zoetermeer, the Netherlands), the cooperative cattle improvement or- ganization CRV BV (Arnhem, the Netherlands), the Dutch Technology Foundation (STW, Utrecht, the Netherlands), the Dutch Ministry of Economic Affairs (The Hague, the Netherlands), and the Provinces of Gelderland and Overijssel (Arnhem, the Netherlands), are thanked for providing the (imputed) 777k genotypes and the FA measurements. The authors acknowledge the herd owners for their help in collecting the data.

\section{REFERENCES}

Astrup, A., J. Dyerberg, P. Elwood, K. Hermansen, F. B. Hu, M. U. Jakobsen, F. J. Kok, R. M. Krauss, J. M. Lecerf, P. LeGrand, P. Nestel, U. Risérus, T. Sanders, A. Sinclair, S. Stender, T. Tholstrup, and W. C. Willett. 2011. The role of reducing intakes of saturated fat in the prevention of cardiovascular disease: Where does the evidence stand in 2010? Am. J. Clin. Nutr. 93:684-688.

Balding, D. J. 2006. A tutorial on statistical methods for population association studies. Nat. Rev. Genet. 7:781-791.

Bernard, L., C. Leroux, and Y. Chilliard. 2013. Expression and nutritional regulation of stearoyl-coa desaturase genes in the ruminant mammary gland: Relationship with milk fatty acid composition. Pages 161-193 in Stearoyl-CoA Desaturase Genes in Lipid Metabolism. P. D. J. M. Ntambi, ed. Springer, New York,

Bouwman, A. C., H. Bovenhuis, M. Visker, and J. van Arendonk. 2011. Genome-wide association of milk fatty acids in Dutch dairy cattle. BMC Genet. 12:43.

Bouwman, A. C., M. H. Visker, J. A. van Arendonk, and H. Bovenhuis. 2012. Genomic regions associated with bovine milk fatty acids in both summer and winter milk samples. BMC Genet. 13:93.

Bouwman, A. C., M. H. P. W. Visker, J. M. van Arendonk, and H. Bovenhuis. 2014. Fine mapping of a quantitative trait locus for bovine milk fat composition on Bos taurus autosome 19. J. Dairy Sci. 97:1139-1149.

Brotherstone, S., and M. Goddard. 2005. Artificial selection and maintenance of genetic variance in the global dairy cow population. Philos. Trans. R. Soc. Lond. B, Biol. Sci. 360:1479-1488.

Browning, S. R., and B. L. Browning. 2007. Rapid and accurate haplotype phasing and missing-data inference for whole-genome association studies by use of localized haplotype clustering. Am. J. Hum. Genet. 81:1084-1097.

de Roos, A. P. W., B. J. Hayes, R. J. Spelman, and M. E. Goddard. 2008. Linkage disequilibrium and persistence of phase in HolsteinFriesian, Jersey and Angus cattle. Genetics 179:1503-1512.

Duchemin, S., H. Bovenhuis, W. Stoop, A. Bouwman, J. Van Arendonk, and M. Visker. 2013. Genetic correlation between composition of bovine milk fat in winter and summer, and DGAT1 and SCD1 by season interactions. J. Dairy Sci. 96:592-604.

Erbe, M., B. J. Hayes, L. K. Matukumalli, S. Goswami, P. J. Bowman, C. M. Reich, B. A. Mason, and M. E. Goddard. 2012. Improving accuracy of genomic predictions within and between dairy cattle breeds with imputed high-density single nucleotide polymorphism panels. J. Dairy Sci. 95:4114-4129.

European Cattle Genetic Diversity Consortium. 2006. Marker-assisted conservation of European cattle breeds: An evaluation. Anim. Genet. 37:475-481.

Gilmour, A. R., B. J. Gogel, B. R. Cullis, and R. Thompson. 2009. ASReml User Guide Release 3.0. VSN International Ltd., Hemel Hempstead, UK.

Goddard, M. E., and B. J. Hayes. 2009. Mapping genes for complex traits in domestic animals and their use in breeding programmes. Nat. Rev. Genet. 10:381-391.

Grisart, B., W. Coppieters, F. Farnir, L. Karim, C. Ford, P. Berzi, N. Cambisano, M. Mni, S. Reid, P. Simon, R. Spelman, M. Georges, and R. Snell. 2002. Positional candidate cloning of a QTL in dairy cattle: Identification of a missense mutation in the bovine DGAT1 gene with major effect on milk yield and composition. Genome Res. 12:222-231. 
Habier, D., R. Fernando, K. Kizilkaya, and D. Garrick. 2011. Extension of the Bayesian alphabet for genomic selection. BMC Bioinformatics 12:186.

Harris, B. L., F. E. Creagh, A. M. Winkelman, and D. L. Johnson. 2011. Experiences with the Illumina high density Bovine BeadChip. Interbull Bull. 44:3-7.

Hayes, B. J., P. Bowman, A. Chamberlain, K. Verbyla, and M. Goddard. 2009. Accuracy of genomic breeding values in multi-breed dairy cattle populations. Genet. Sel. Evol. 41:51.

Hozé, C., S. Fritz, F. Phocas, D. Boichard, V. Ducrocq, and P. Croiseau. 2014. Efficiency of multi-breed genomic selection for dairy cattle breeds with different sizes of reference population. J. Dairy Sci. 97:3918-3929.

ISO-IDF. 2002a. Milkfat - Determination of the fatty acid composition by gas-liquid chromatography. ISO 15885-IDF 184 . International Dairy Federation, Brussels, Belgium.

ISO-IDF. 2002b. Milkfat-Preparation of fatty acid methyl ester. ISO 15884-IDF 184. International Dairy Federation, Brussels, Belgium.

Kemper, K. E., and M. E. Goddard. 2012. Understanding and predicting complex traits: Knowledge from cattle. Hum. Mol. Genet. 21:R45-R51.

Kgwatalala, P. M., P. M. Kgwatalala, E. M. Ibeagha-Awemu, P. M. Kgwatalala, E. M. Ibeagha-Awemu, J. F. Hayes, P. M. Kgwatalala, E. M. Ibeagha-Awemu, J. F. Hayes, and X. Zhao. 2007. Single nucleotide polymorphisms in the open reading frame of the stearoyl-CoA desaturase gene and resulting genetic variants in Canadian Holstein and Jersey cows. DNA Seq. 18:357-362.

Maurice-Van Eijndhoven, M. H. T., H. Bovenhuis, H. Soyeurt, and M. P. L. Calus. 2013a. Differences in milk fat composition predicted by mid-infrared spectrometry among dairy cattle breeds in the Netherlands. J. Dairy Sci. 96:2570-2582.

Maurice-Van Eijndhoven, M. H. T., S. J. Hiemstra, and M. P. L. Calus. 2011. Short communication: Milk fat composition of 4 cattle breeds in the Netherlands. J. Dairy Sci. 94:1021-1025.

Maurice-Van Eijndhoven, M. H. T., H. Soyeurt, F. Dehareng, and M. P. L. Calus. 2013b. Validation of fatty acid predictions in milk using mid-infrared spectrometry across cattle breeds. Animal $7: 348-354$.

Maurice-Van Eijndhoven, M. H. T., R. F. Veerkamp, H. Soyeurt, and M. P. L. Calus. 2014. Heritability of milk fat composition is considerably lower for Meuse-Rhine-Yssel cows compared to Holstein Friesian. Pages 83-102 in Genetic Variation of Milk Fatty Acid Composition Between and Within Dairy Cattle Breeds. M. H. T. Maurice-Van Eijndhoven, PhD Thesis. Wageningen University, Wageningen, the Netherlands.

Mele, M., G. Conte, B. Castiglioni, S. Chessa, N. Macciotta, A. Serra, A. Buccioni, G. Pagnacco, and P. Secchiari. 2007. Stearoyl-coenzyme A desaturase gene polymorphism and milk fatty acid composition in Italian Holsteins. J. Dairy Sci. 90:4458-4465.

Oldenbroek, K. (Editor) 2007. Utilisation and Conservation of Farm Animal Genetic Resources. Wageningen Academic Publishers, Wageningen, the Netherlands.

Palmquist, D. L., K. Stelwagen, and P. H. Robinson. 2006. Modifying milk composition to increase use of dairy products in healthy diets. Anim. Feed Sci. Technol. 131:149-153.

Pryce, J. E., B. Gredler, S. Bolormaa, P. J. Bowman, C. Egger-Danner, C. Fuerst, R. Emmerling, J. Sölkner, M. E. Goddard, and B.
J. Hayes. 2011. Short communication: Genomic selection using a multi-breed, across-country reference population. J. Dairy Sci. 94:2625-2630.

Rutten, M. J. M., H. Bovenhuis, K. A. Hettinga, H. J. F. van Valenberg, and J. A. M. van Arendonk. 2009. Predicting bovine milk fat composition using infrared spectroscopy based on milk samples collected in winter and summer. J. Dairy Sci. 92:6202-6209.

Schennink, A., J. Heck, H. Bovenhuis, M. Visker, H. van Valenberg, and J. van Arendonk. 2008a. Milk fatty acid unsaturation: Genetic parameters and effects of stearoyl-CoA desaturase (SCD1) and acyl CoA: diacylglycerol acyltransferase 1 (DGAT1). J. Dairy Sci. 91:2135-2143

Shook, G. E. 2006. Major advances in determining appropriate selection goals. J. Dairy Sci. 89:1349-1361.

Smet, K., J. De Block, S. De Campeneere, D. De Brabander, L. Herman, K. Raes, K. Dewettinck, and K. Coudijzer. 2009. Oxidative stability of UHT milk as influenced by fatty acid composition and packaging. Int. Dairy J. 19:372-379.

Soyeurt, H., P. Dardenne, A. Gillon, C. Croquet, S. Vanderick, P. Mayeres, C. Bertozzi, and N. Gengler. 2006. Variation in fatty acid contents of milk and milk fat within and across breeds. J. Dairy Sci. 89:4858-4865.

Soyeurt, H., F. Dehareng, N. Gengler, S. McParland, E. Wall, D. P. Berry, M. Coffey, and P. Dardenne. 2011. Mid-infrared prediction of bovine milk fatty acids across multiple breeds, production systems, and countries. J. Dairy Sci. 94:1657-1667.

Soyeurt, H., F. Dehareng, P. Mayeres, C. Bertozzi, and N. Gengler. 2008. Variation of $\Delta 9$-desaturase activity in dairy cattle. J. Dairy Sci. 91:3211-3224.

Spelman, R. J., C. A. Ford, P. McElhinney, G. C. Gregory, and R. G. Snell. 2002. Characterization of the DGAT1 gene in the New Zealand dairy population. J. Dairy Sci. 85:3514-3517.

Stoop, W. M., J. van Arendonk, J. Heck, H. van Valenberg, and H. Bovenhuis. 2008. Genetic parameters for major milk fatty acids and milk production traits of Dutch Holstein-Friesians. J. Dairy Sci. 91:385-394.

Thaller, G., W. Krämer, A. Winter, B. Kaupe, G. Erhardt, and R. Fries. 2003. Effects of DGAT1 variants on milk production traits in German cattle breeds. J. Anim. Sci. 81:1911-1918.

Verbyla, K. L., M. P. L. Calus, H. A. Mulder, Y. de Haas, and R. F. Veerkamp. 2010. Predicting energy balance for dairy cows using high-density single nucleotide polymorphism information. J. Dairy Sci. 93:2757-2764.

Wang, X., C. Wurmser, H. Pausch, S. Jung, F. Reinhardt, J. Tetens, G. Thaller, and R. Fries. 2012. Identification and dissection of four major QTL affecting milk fat content in the German Holstein-Friesian population. PLoS ONE 7:e40711. http://dx.doi. org/10.1371/journal.pone.0040711.

Wiggans, G. R., T. S. Sonstegard, P. M. Vanraden, L. K. Matukumalli, R. D. Schnabel, J. F. Taylor, F. S. Schenkel, and C. P. Van Tassell. 2009. Selection of single-nucleotide polymorphisms and quality of genotypes used in genomic evaluation of dairy cattle in the United States and Canada. J. Dairy Sci. 92:3431-3436.

Wilmink, J. B. M. 1987. Adjustment of test-day milk, fat and protein yield for age, season and days-in-milk. Livest. Prod. Sci. $16: 335-348$. 